DESY 08-128

TUM-HEP 699/08

\title{
Hidden gauginos of an unbroken $U(1)$ : Cosmological constraints and phenomenological prospects
}

\author{
A. Ibarra ${ }^{a, b}$, A. Ringwald ${ }^{b}$, C. Weniger $^{b}$ \\ a Physik Department T30, Technische Universität München, \\ James-Franck-Strasse, 85748 Garching, Germany. \\ $b$ Deutsches Elektronen-Synchrotron DESY, \\ Notkestrasse 85, 22607 Hamburg, Germany.
}

\begin{abstract}
We study supersymmetric scenarios where the dark matter is the gaugino of an unbroken hidden $U(1)$ which interacts with the visible world only via a small kinetic mixing with the hypercharge. Strong constraints on the parameter space can be derived from avoiding overclosure of the Universe and from requiring successful Big Bang Nucleosynthesis and structure formation. We find that for typical values of the mixing parameter, scenarios with neutralino NLSP are excluded, while scenarios with slepton NLSP are allowed when the mixing parameter lies in the range $\chi \sim \mathcal{O}\left(10^{-13}-10^{-10}\right)$. We also show that if the gravitino is the LSP and the hidden $U(1)$ gaugino the NLSP, the bounds on the reheating temperature from long lived charged MSSM relics can be considerably relaxed and we comment on the signatures of these scenarios at future colliders. Finally, we discuss the case of an anomalously small mixing, $\chi \ll 10^{-16}$, where the neutralino becomes a decaying dark matter candidate, and derive constraints from gamma ray experiments.
\end{abstract}




\section{Introduction}

The existence of dark matter in the Universe is perhaps the most solid indication for physics beyond the Standard Model of particle physics [1]. Among the many extensions of the Standard Model that have been proposed in recent years, supersymmetry (SUSY) arguably remains as the most popular. Among other merits, supersymmetric scenarios provide a very promising candidate for the dark matter: the lightest supersymmetric particle (LSP) [2, 3]. With the particle content of the Minimal Supersymmetric Standard Model (MSSM), the LSP can be, over a large range of parameters, either the lightest neutralino, the lightest sneutrino or the lightest stau. Among these, only the lightest neutralino is still allowed by present experiments as a viable dark matter candidate, provided $R$-parity is almost exactly conserved. Furthermore, if supersymmetry is promoted to a local symmetry, the particle content of the MSSM also includes the gravitational supermultiplet, of which the spin $3 / 2$ component, the gravitino, is also a viable dark matter candidate [4, even if $R$-parity is slightly violated [5, 6].

On the other hand, many extensions of the MSSM contemplate the possibility of a hidden sector, consisting of superfields which are singlets under the Standard Model gauge group. Hidden sector superfields usually couple very weakly to our observable sector, thus constituting a very natural arena for finding dark matter candidates. Generically, hidden sector particles couple to our observable sector only via non-renormalizable operators, presumably suppressed by a large mass scale, with a structure that is strongly model dependent. In consequence, deriving implications of the hidden sector dark matter on the thermal history of the observable Universe and for future collider experiments is hindered by our complete ignorance of the strength and the structure of the hidden sector interactions with our observable sector.

There are however three instances where the hidden sector particles can couple to the MSSM particles via renormalizable operators, with a structure which is well defined by the Lorentz and gauge symmetries. Firstly, a hidden sector chiral superfield, $S$, could couple to the lepton and up-type Higgs doublet superfields via the Yukawa coupling $S H_{u} L$ in the superpotential, or to the two MSSM Higgs doublets, via $S_{u} H_{d}$, provided these terms are also invariant under the hidden sector gauge group (as well as possible discrete and global symmetries of the theory). Secondly, if one of the MSSM chiral superfields is charged under a hidden sector gauge group, it will interact with the corresponding hidden sector gauge superfield, and in turn with other hidden sector chiral superfields via the $D$-term. Finally, a hidden sector abelian vector superfield, $X$, may couple to the hypercharge vector 
superfield through a kinetic mixing term, which is always allowed by the gauge symmetries [7, 8].

In this paper we will concentrate on the last situation and we will study scenarios where the hidden $U(1)$ gauge group is unbroken at low energies. If this is the case, the corresponding hidden $U(1)$ gaugino will have a mass comparable to the typical soft SUSY breaking masses of the observable sector particles and, in some instances, smaller. Therefore, the hidden $U(1)$ gaugino will not only be the lightest supersymmetric particle of the hidden sector, but also the lightest among all the supersymmetric particles.

Our motivation to consider an unbroken hidden sector $U(1)$ group is twofold. First, in string theory compactifications hidden sector $U(1)$ groups are ubiquitous and some of them could remain unbroken at low energies, in complete analogy to the familiar electromagnetic $U(1)$ of our observable sector. Secondly, the case of the hidden sector unbroken $U(1)$ is particularly intriguing, since this situation is practically unconstrained by present experiments. Indeed, it was shown long ago by Holdom that, in a nonsupersymmetric world, the hidden $U(1)$ gauge boson (the "paraphoton" [9]) completely decouples from the observable sector [10]. This result can be also generalized to a supersymmetric theory. Let us consider the SUSY invariant part of the Lagrangian,

$$
\begin{aligned}
\mathcal{L}= & \int d^{2} \theta\left(\hat{W}_{B}^{\alpha} \hat{W}_{B \alpha}+\hat{W}_{X}^{\alpha} \hat{W}_{X \alpha}+2 \chi \hat{W}_{B}^{\alpha} \hat{W}_{X \alpha}\right)+\text { h.c. }+ \\
& +\int d^{2} \theta d^{2} \bar{\theta}\left(\Phi^{\dagger} e^{Y g_{Y} \hat{B}} \Phi+h^{\dagger} e^{q g_{X} \hat{X}_{h}} h\right)
\end{aligned}
$$

where the field strength superfield is defined as $\hat{W}_{V}^{\alpha}=-\frac{1}{4} \bar{D} \bar{D} D^{\alpha} \hat{V}, \hat{V}=$ $\hat{B}, \hat{X}$ being the hypercharge or the hidden $U(1)$ vector superfield, while $\Phi$ and $h$ denote, respectively, any Standard Model or hidden sector chiral superfield. Finally, $\chi$ is the kinetic mixing parameter, which is induced through quantum effects by chiral superfields charged under both gauge groups. Without additional symmetries, values around $\chi \sim 10^{-3}-10^{-4}$ are naturally obtained. However, e.g. in compactifications of heterotic [11, 12] and type II [13, 14, 15, 16, 17] strings, much smaller mixings are possible. A lower bound around $\chi \gtrsim 10^{-16}$ was argued to hold in cases of gauge mediated supersymmetry breaking in heterotic string models [11], whereas in type II models with warped extra dimensions the kinetic mixing parameter can be exponentially small [17].

The gauge kinetic terms in Eq. (11) can be made canonical by introducing 
shifted vector superfields,

$$
\begin{aligned}
X & =\hat{X}+\chi \hat{B} \\
B & =\sqrt{1-\chi^{2}} \hat{B},
\end{aligned}
$$

leading to

$$
\begin{aligned}
\mathcal{L}= & \int d^{2} \theta\left(W_{B}^{\alpha} W_{B \alpha}+W_{X}^{\alpha} W_{X \alpha}\right)+\text { h.c. }+ \\
& +\int d^{2} \theta d^{2} \bar{\theta}\left(\Phi^{\dagger} e^{Y g_{Y}^{\prime} B} \Phi+h^{\dagger} e^{q g_{X} X-q g_{X}^{\prime} B} h\right),
\end{aligned}
$$

where $g_{Y}^{\prime}=g_{Y} / \sqrt{1-\chi^{2}}$ and $g_{X}^{\prime}=\chi g_{X} / \sqrt{1-\chi^{2}}$. Therefore, the canonical normalization of the kinetic terms produces an unobservable shift of the hypercharge gauge coupling and the generation of a "minihypercharge" for the hidden sector chiral superfields [7]. Different astrophysical observations and laboratory experiments constrain the possible values of the minihypercharge and the masses of the hidden sector particles. For instance, one obtains $\chi \lesssim 10^{-13}$ for masses below $10 \mathrm{keV}$ from plasmon decay in red giants [18, 19] (see also Ref. [20]). Nevertheless these bounds can be automatically avoided if the masses are large. In this case, as long as supersymmetry remains unbroken, the hypercharge vector superfield completely decouples from the observable sector and is not subject to any experimental constraint.

The breaking of supersymmetry changes dramatically the previous picture. Although the hidden $U(1)$ gauge boson remains decoupled from the observable sector, we will show that in the presence of SUSY breaking effects a mixing between the hidden $U(1)$ gaugino and the MSSM neutralinos is induced. Then, the unbroken hidden $U(1)$ might produce observable effects in the cosmological evolution of the Universe, at collider experiments or in cosmic ray fluxes.

Models with a hidden $U(1)$ extension of the SM or MSSM have been extensively studied in the literature (see e.g. [21, for a recent review see Ref. [22]). Some of these works take into account kinetic- and mass-mixing $[23,24,25,26,27,28,29$, but it is typically assumed that the gauge symmetry of the additional $U(1)$ is broken by a Higgs or Stueckelberg mechanism 1 In this case, it is possible to derive bounds from high precision LEPI data

\footnotetext{
${ }^{1}$ Exceptions are e.g. Ref. [30, 31], where constraints on, and consequences of, higher dimensional operators that couple hidden and observable sector are studied. In Ref. 32] the authors study BBN and CMB constraints on the particle content of a completely decoupled hidden sector which may contain unbroken $U(1)$ s. Furthermore, see Ref. 33 for a short discussion about gauge coupling unification in the presence of kinetic mixing.
} 
if the hidden $U(1)$ gauge boson mass is large (namely, $\chi \lesssim 0.05$ for masses around $200 \mathrm{GeV}$ [24]), and from different astronomical observations and laboratory experiments if the masses are small (namely, for masses around $100 \mathrm{eV}$, the observed lifetime of the sun translates into a bound $\chi \lesssim 10^{-13}$ 34, see Ref. 35] for bounds from considerations of the hidden CMB).

The purpose of this paper is to consider in some detail cosmological constraints and phenomenological properties of a kinetically mixed hidden $U(1)$ extension of the MSSM with unbroken gauge symmetry. We will assume that the hidden $U(1)$ gaugino is the LSP in most of the paper, and concentrate on its prospect of being dark matter. In section 2, we will describe the model in the component formalism and discuss typical values for mass- and mixing-parameters in scenarios with gauge and gravity mediation of supersymmetry breaking. In section 3, we derive a bound on the mixing parameter from thermal overproduction of the hidden $U(1)$ gaugino. Section 4 considers bounds from primordial nucleosynthesis for the cases of stau and neutralino NLSPs. There, we also analyze the effects of a possibly light gravitino and show how bounds on the reheating temperature can be relaxed. In section 5 we briefly discuss the collider phenomenology of this scenario. In section 6 we study the case with an anomalously small kinetic mixing, where the neutralino NLSP could become a viable, though unstable, dark matter candidate. Finally, we present our conclusions and an outlook in section 7 .

\section{Model}

In this section, we will briefly describe the model under consideration in the more familiar component formalism. In the Wess-Zumino gauge, a vector superfield can be expanded in component fields as $V=-\theta \sigma^{\mu} \bar{\theta} V_{\mu}+i \theta \theta \bar{\theta} \bar{\lambda}-$ $i \bar{\theta} \bar{\theta} \theta \lambda+\frac{1}{2} \theta \theta \bar{\theta} \bar{\theta} D$. Then, the pure gauge part of Eq. (11), including supersymmetry breaking effects, reads:

$$
\begin{aligned}
\mathcal{L}_{\text {gauge }}= & -\frac{1}{4}\left(\begin{array}{ll}
\hat{X}_{\mu \nu} & \hat{B}_{\mu \nu}
\end{array}\right) \mathcal{K}\left(\begin{array}{l}
\hat{X}^{\mu \nu} \\
\hat{B}^{\mu \nu}
\end{array}\right)-i\left(\begin{array}{ll}
\hat{\lambda}_{X}^{\dagger} & \hat{\lambda}_{B}^{\dagger}
\end{array}\right) \mathcal{K} \bar{\sigma}^{\mu} \partial_{\mu}\left(\begin{array}{l}
\hat{\lambda}_{X} \\
\hat{\lambda}_{B}
\end{array}\right) \\
& +\frac{1}{2}\left(\begin{array}{ll}
\hat{D}_{X}^{*} & \hat{D}_{B}^{*}
\end{array}\right) \mathcal{K}\left(\begin{array}{l}
\hat{D}_{X} \\
\hat{D}_{B}
\end{array}\right)-\frac{1}{2}\left(\begin{array}{ll}
\hat{\lambda}_{X} & \hat{\lambda}_{B}
\end{array}\right) \hat{\mathcal{M}}\left(\begin{array}{l}
\hat{\lambda}_{X} \\
\hat{\lambda}_{B}
\end{array}\right)+\text { h.c. },
\end{aligned}
$$

where $\mathcal{K}$ and $\hat{\mathcal{M}}$ denote, respectively, the kinetic and mass mixing matrices:

$$
\mathcal{K}=\left(\begin{array}{ll}
1 & \chi \\
\chi & 1
\end{array}\right) \quad \text { and } \quad \hat{\mathcal{M}}=\left(\begin{array}{ll}
\hat{M}_{X} & \delta \hat{M} \\
\delta \hat{M} & \hat{M}_{B}
\end{array}\right)
$$


It is convenient to work in the basis where the kinetic terms are canonical. To this end, we will redefine the vector superfields according to

$$
\left(\begin{array}{l}
\hat{X} \\
\hat{B}
\end{array}\right)=\left(\begin{array}{cc}
1 & -\frac{\chi}{\sqrt{1-\chi^{2}}} \\
0 & \frac{1}{\sqrt{1-\chi^{2}}}
\end{array}\right)\left(\begin{array}{l}
X \\
B
\end{array}\right) .
$$

As explained in the introduction, the canonically normalized fields $X_{\mu \nu}$ and $D_{X}$ completely decouple from the observable sector. On the other hand, a mixing between the hidden gaugino and the bino remains, unless $\delta \hat{M} \simeq$ $\chi \hat{M}_{X}$. More precisely, in the basis where the kinetic terms are canonical, the extended neutralino $(5 \times 5)$ mass matrix reads, to lowest order in $\chi$,

$$
\mathcal{M}_{\mathrm{N}}=\left(\begin{array}{ccccc}
M_{X} & \delta M & 0 & 0 & 0 \\
\delta M & M_{B} & 0 & -M_{Z} c_{\beta} s_{W} & M_{Z} s_{\beta} s_{W} \\
0 & 0 & M_{W} & M_{Z} c_{\beta} c_{W} & -M_{Z} s_{\beta} c_{W} \\
0 & -M_{Z} c_{\beta} s_{W} & M_{Z} c_{\beta} c_{W} & 0 & -\mu \\
0 & M_{Z} s_{\beta} s_{W} & -M_{Z} s_{\beta} c_{W} & -\mu & 0
\end{array}\right),
$$

where, $\delta M \simeq \delta \hat{M}-\chi \hat{M}_{X}, M_{X} \simeq \hat{M}_{X}$ and $M_{B} \simeq \hat{M}_{B}$. Here, $\mu$ denotes the MSSM $\mu$-term, $M_{Z}$ the mass of the $Z^{0}$ gauge boson, $s_{W}$ the sine of the Weinberg angle and $s_{\beta}$ is related to the ratio of the two Higgs VEVs.

Lastly, as was mentioned in the introduction, hidden sector matter particles charged under the hidden $U(1)$ will acquire a minihypercharge, as a consequence of the canonical normalization of the kinetic terms. For simplicity we will assume throughout the paper that hidden sector matter particles are too heavy to be produced during reheating, and thus do not influence the evolution of the Universe.

The size of the gaugino mass terms $\hat{\mathcal{M}}$ in the Lagrangian, Eq.(15), is very model dependent. For example, in models with gravity mediation, gaugino masses would arise from the term

$$
\mathcal{L} \supset \int d \theta^{2} d \bar{\theta}^{2} \frac{1}{M_{P}}\left(\hat{Z}_{1}^{\dagger} \hat{W}_{B} \hat{W}_{B}+2 \chi \hat{Z}_{2}^{\dagger} \hat{W}_{B} \hat{W}_{X}+\hat{Z}_{3}^{\dagger} \hat{W}_{X} \hat{W}_{X}\right) \text {. + h.c. }
$$

where coefficients of order one have been dropped. In this equation $\hat{Z}_{i}$ are three spurion superfields, which will eventually acquire a vacuum expectation value, $\left\langle\hat{Z}_{i}\right\rangle=M_{P}+\theta^{2} F_{i}$, by some unspecified mechanism 2, yielding

\footnotetext{
${ }^{2}$ We assume that breaking of supersymmetry takes place in a sector that is distinct from the hidden $U(1)$ under consideration, hence the $D$-terms are zero and kinetic mixing has no effect on the MSSM mass spectrum (like e.g. in Ref. [1]).
} 
$\hat{M}_{B}=F_{1} / M_{P}, \delta \hat{M}=\chi F_{2} / M_{P}, \hat{M}_{X}=F_{3} / M_{P}$, where $M_{P} \simeq 2.4 \times 10^{18} \mathrm{GeV}$ denotes the reduced Planck mass.

The simplest case consists on assuming just one spurion superfield which couples universally to all vector superfields, namely $Z_{i} \equiv Z$ for all $i$. If this is the case, at the scale $M_{P}$, the different soft terms satisfy the relations $\hat{M}_{B}=\hat{M}_{X}=\sqrt{3} m_{3 / 2}$ and $\delta \hat{M}=\sqrt{3} \chi m_{3 / 2}, m_{3 / 2}$ being the gravitino mass. Therefore, after the canonical normalization of the gaugino kinetic terms, the mass mixing term $\delta M$ in Eq. (8) vanishes. However, the universality of the gaugino mass terms is broken by quantum effects, thus inducing a nonvanishing $\delta M$ at low energies. Using the renormalization group equations for the gaugino mass matrix in appendix $\mathrm{B}$, we find that the mass mixing at the electroweak scale reads

$$
\delta M_{\mathrm{EW}} \simeq \frac{1}{8 \pi^{2}} g_{X}^{2} B_{X X} \ln \left(\frac{M_{P}}{M_{\mathrm{hid}}}\right) \chi_{\mathrm{EW}} M_{X}
$$

where $g_{X}$ is the gauge coupling of the hidden $U(1)$ gauge group, $M_{\text {hid }}$ is the mass scale of hidden sector matter, and $B_{X X}=\sum_{i} Q_{i}^{2}$ is the sum, for all the particles in the theory, of all the hidden $U(1)$ charges squared. On the other hand, the renormalization group evolution will also make the hidden gaugino mass smaller at low energies. If there is an enough number of (heavy) matter states in the hidden sector, the hidden gaugino will become the lightest supersymmetric particle.

In more general scenarios with gravity mediation, where several spurion fields contribute to the breaking of supersymmetry, a tree level mixing between the bino and the hidden gaugino will usually remain after canonical normalization of the kinetic terms. On the other hand, the nature of the lightest supersymmetric particle, whether it is the gravitino, the bino or the hidden gaugino, depends on the details of the model.

The most natural scenarios with a light hidden $U(1)$ gaugino are probably those with gauge mediated supersymmetry breaking. Assuming that the messenger fields are not charged under the hidden $U(1)$ gauge group, the soft mass of the hidden gaugino will entirely come from gravity effects. If this is the case, the gravitino and the hidden $U(1)$ gaugino will acquire similar masses, which are naturally much smaller than the masses of the supersymmetric particles of the MSSM. Again, whether the LSP is the gravitino or the hidden gaugino depends on the particle content of the hidden sector, which will drive the hidden gaugino mass to smaller values at low energies through radiative effects.

An important quantity in our analysis will be the mixing angle $\Theta$ between 
the bino and the hidden $U(1)$ gaugino mass and interaction eigenstates 3 . The approximate diagonalization of Eq. (8) yields

$$
\Theta \simeq \frac{\delta M^{\mathrm{EW}}}{M_{B}^{\mathrm{EW}}-M_{X}^{\mathrm{EW}}},
$$

where we have emphasized that all the quantities should be evaluated at the electroweak scale. In the scenarios of supersymmetry breaking proposed in this section with hidden $U(1)$ gaugino as LSP, the mixing angle $\Theta$ can be written as

$$
\Theta \simeq C \cdot \chi_{\mathrm{EW}} \frac{M_{X}^{\mathrm{EW}}}{M_{B}^{\mathrm{EW}}-M_{X}^{\mathrm{EW}}}
$$

where $C$ is a constant that can roughly vary between $10^{-2} \lesssim C \lesssim 1$, depending on whether $\delta M^{\mathrm{EW}}$ is already present at tree level or is generated radiatively, and on the particle content of the hidden sector. For definiteness we will choose $C=1$ in the subsequent analysis.

Following the above discussion, we will first study the phenomenology of a hidden $U(1)$ gaugino LSP, and second the case where both, the hidden $U(1)$ gaugino and the gravitino, constitute the lightest supersymmetric particles.

\section{Overproduction constraints}

The least model dependent bound on the hidden $U(1)$ gaugino scenario comes from considerations of the thermal history of the universe. After reheating of the visible sector, hidden $U(1)$ gauginos can be produced in the hot primeval plasma through the mixing with the MSSM neutralinos.

This mechanism is similar to the thermal production of gravitinos or axions (see e.g. Ref. [36, 37, 38]). However, in contrast to these cases, the final abundance of the hidden $U(1)$ gaugino does not depend on the reheating temperature, $T_{R}$. This follows from the fact that mass- and kinetic-mixings appear at the renormalizable level of the Lagrangian, whereas gravitino and axion couplings are mass-suppressed. Actually, the production is most efficient at low temperatures, and this makes an exact calculation of the hidden $U(1)$ gaugino abundance extremely complicated, since at temperatures around $T \sim 100 \mathrm{GeV}$ the QCD coupling constant $g_{s}$ is of the order of one, which precludes a sharp separation between hard, $T$, and soft, $g_{s} T$, momenta as required for the proper treatment of hard thermal loops and screening

\footnotetext{
${ }^{3}$ Note that we always assume the mass hierarchy $M_{B}<M_{W}<\mu$ in this paper.
} 


\begin{tabular}{|l|c|}
\hline Process & $\overline{|\mathcal{M}|^{2}} / \Theta^{2}\left(g^{\prime} g_{s}\right)^{2}\left|T_{b a}^{j}\right|^{2} Y_{L, R}^{2}$ \\
\hline \hline 1) $q g \rightarrow \tilde{q} \lambda_{X}$ & $-2 \frac{u}{s}$ \\
\hline 2) $\tilde{q} \tilde{g} \rightarrow \tilde{q} \lambda_{X}$ & $-2\left(\frac{t}{s}+\frac{s}{t}\right)$ \\
\hline 3) $\tilde{q} g \rightarrow q \lambda_{X}$ & $2 \frac{u}{t}$ \\
\hline 4) $q \tilde{g} \rightarrow q \lambda_{X}$ & 4 \\
\hline 5) $q \tilde{q} \rightarrow g \lambda_{X}$ & $-4 \frac{s}{u}$ \\
\hline 6) $q \bar{q} \rightarrow \tilde{g} \lambda_{X}$ & 8 \\
\hline 7) $\tilde{q} \tilde{q} \rightarrow \tilde{g} \lambda_{X}$ & $4\left(\frac{t}{u}+\frac{u}{t}\right)$ \\
\hline
\end{tabular}

Table 1: Squared matrix elements for inelastic two-body scatterings with a hidden $U(1)$ gaugino in the final state. Here, $T_{b a}^{i}$ denotes the generators of the fundamental representation of the $S U(3)$ gauge group, $Y_{L, R}$ the hypercharges of the (s)quarks, and $g^{\prime}$ and $g_{s}$ the gauge couplings of the electroweak and strong interactions, respectively. We average over initial and sum over final spins.

effects [39. How to deal with this drawback is an open problem even in the milder case of gravitino production (see, however, Ref. [38]).

For the purposes of this paper it is enough to derive an order of magnitude estimate on the hidden $U(1)$ gaugino abundance. To this end, we have calculated the collision integral in a relativistic QCD plasma, taking into account $2 \rightarrow 2$ scattering in the QCD and hypercharge sector with a bino in the final state. Since binos mix with hidden $U(1)$ gauginos with the mixing angle $\Theta$, the production rate of hidden $U(1)$ gauginos can be straightforwardly calculated by multiplying the result for the binos by $\Theta^{2}$. The relevant scattering processes and the corresponding squared matrix elements are listed in Tab. 1.

Summing over all spins and particles, the collision term of hidden $U(1)$ gauginos in the relativistic MSSM plasma reads 4

$$
\begin{aligned}
\gamma_{\mathrm{QCD}} \equiv \frac{d^{4} n_{X}}{d V d t} & =\frac{308}{3 \pi^{3}} \alpha^{\prime} \alpha_{s} \Theta(T)^{2}\left(1-\frac{4}{7} \gamma_{E}-\frac{4}{7} \ln \frac{k^{*}}{T}\right) T^{4} \\
& \simeq 3 \times 10^{-3} \Theta(T)^{2} T^{4}
\end{aligned}
$$

where one has in general to take into account the temperature dependence of the mixing angle $\Theta$, which stems from the thermal mass of the bino (see Eq. (12)). Here, we simply take $M_{B}(T) \simeq M_{B}$, since most of the production is expected to happen when the particles become non-relativistic. In this

\footnotetext{
${ }^{4}$ We use Boltzmann distribution functions for simplicity.
} 
equation, $\alpha^{\prime}$ and $\alpha_{s}$ denote the hypercharge and QCD couplings, respectively, and $\gamma_{E} \simeq 0.577$. Furthermore, $k^{*}$ denotes the cut-off of intermediate three-momenta in $t$-channel processes. Only processes with quarks in the intermediate state contribute to these divergences, and we identify $k^{*}$ with their thermal mass (at $T \sim 100 \mathrm{GeV}$ the thermal quark masses lie between $0.63 T$ and $0.84 T$ [38]). Finally, solving the Boltzmann equation yields the thermal abundance of hidden $U(1)$ gauginos,

$$
\Omega_{X} h^{2} \approx 5.5 \times 10^{7}\left(\frac{M_{X}}{100 \mathrm{GeV}}\right) \int_{T_{0}}^{T_{R}} d T \frac{M_{P}}{T^{2}} \frac{\gamma_{\mathrm{QCD}}(T) \Theta^{2}(T)}{T^{4}}
$$

Taking into account thermal masses could actually lead to a further orderone enhancement due to particle decay in the QCD plasma 38. Contributions from electroweak interactions and Yukawa couplings are also expected to give sizable corrections, again of order one.

Requiring that $\Omega_{X} h^{2} \lesssim 0.1$ gives the overproduction bound

$$
\Theta \lesssim 5 \times 10^{-12}\left(\frac{M_{X}}{M_{B}}\right)^{-1 / 2},
$$

where we have used $T_{0} \simeq M_{B}$ as a cutoff in Eq. (14), which corresponds to squark- and gluino masses around $3 M_{B}$. For other values the bound scales roughly like $\sqrt{T_{0}}$.

In Fig. 1 (for $M_{B}=180 \mathrm{GeV}$ ) and Fig. 2 (for $M_{B}=150 \mathrm{GeV}$ ) we show the region in the $\left(\chi, M_{X}\right)$ parameter space where the hidden $U(1)$ gaugino is overproduced. Remarkably, a large part of the parameter space suggested by string theory is excluded by the constraint Eq. (15) 5

\section{Bounds from primordial nucleosynthesis and structure formation}

In general, dark matter can be produced thermally and/or in the late decay of some other relic particle $\xi$. The abundance of dark matter today is then given by $\Omega_{\mathrm{DM}}=\Omega_{\mathrm{DM}}^{\mathrm{th}}+\left(M_{\mathrm{DM}} / M_{\xi}\right) \Omega_{\xi}^{\text {th. }}$, where $\Omega_{\xi}^{\text {th. }}$ refers to the thermal abundance of the particle $\xi$. Typical candidates for $\xi$, like the stau or a neutralino, naturally freeze out with an abundance of the right order of magnitude to account for the observed dark matter abundance, and a given

\footnotetext{
${ }^{5}$ A sizable annihilation rate between hidden $U(1)$ gauginos would allow to circumvent this bound. If the mixing angle $\Theta$ is small, this would require additional light matter states in the hidden sector, which do not exist in our scenario.
} 


\begin{tabular}{|c|c|c|c|c|}
\hline & $i)$ & $i i)$ & $i i i)$ & $i v)$ \\
\hline \hline NNLSP & $\cdot$ & $\cdot$ & slepton & slepton \\
NLSP & slepton & neutralino & gravitino & $\lambda_{X}$ \\
LSP & $\lambda_{X}$ & $\lambda_{X}$ & $\lambda_{X}$ & gravitino \\
\hline
\end{tabular}

Table 2: The different scenarios that we will investigate in this work.

dark matter candidate can inherit this property as long as its direct thermal production is negligible and provided the masses of the two particles are comparable.

These so-called superWIMP scenarios (super-weakly interacting massive particles, see Ref. [4]), where the dark matter superWIMPs are produced in the late decay of other particles, are subject to strong constraints due to their potential impact on primordial nucleosynthesis and structure formation. If the production takes place during or after Big Bang Nucleosynthesis (BBN), the hadronic and electromagnetic energy released in the decay can destroy the successful predictions of the standard scenario (see e.g. Ref. [40, 43]). In cases where the decaying particle is electrically charged, it could additionally form bound states with ${ }^{4} \mathrm{He}$, triggering the catalytic production of ${ }^{6} \mathrm{Li}$ and ${ }^{9} \mathrm{Be}$ [41, 44]. Furthermore, the superWIMP itself is potentially produced with a momentum that is large enough to wash out density fluctuations on small scales with impact on structure formation, similar to scenarios with warm dark matter (for a discussion in the context of superWIMPs see Ref. 45]).

Below, we will apply these bounds to the case of a hidden $U(1)$ gaugino superWIMP, concentrating on the four different scenarios shown in Tab. 2 , Following the superWIMP paradigm, the relic abundance of the neutralino or the slepton is fixed by the requirement that the LSP constitutes all dark matter today.

Scenario i) After freeze out, the slepton NLSP decays predominantly through the channel $\tilde{l} \rightarrow \lambda_{X} l$ into a lepton and the hidden $U(1)$ gaugino LSP. The corresponding decay width is given by

$$
\Gamma_{\tilde{l} \rightarrow \lambda_{X} l} \simeq \frac{g^{\prime 2}}{8 \pi} \Theta^{2} Y_{\tilde{l}}^{2} M_{\tilde{l}}\left(1-\frac{M_{X}^{2}}{M_{\tilde{l}}^{2}}\right)^{2},
$$

where $Y_{\tilde{l}}$ and $M_{\tilde{l}}$ denote, respectively, the hypercharge and the mass of the slepton NLSP. 
The decay induces electromagnetic cascades in the plasma, the branching fraction into electromagnetic energy being close to one, $B_{\mathrm{EM}}^{\tilde{l}} \simeq 1$. The corresponding energy release is given by $\epsilon_{\mathrm{EM}}^{\tilde{l}} \approx\left(M_{\tilde{l}}^{2}-M_{X}^{2}\right) /\left(2 M_{\tilde{l}}\right)$ (see Ref. 46] for an exhaustive discussion).

Contributions to the hadronic energy release stem, if kinematically allowed, from three-body decays into $Z^{0}, W^{ \pm}$and Higgs bosons. The corresponding branching ratios are all of the order of, or smaller than, $\alpha^{\prime} /\left(16 \pi s_{\mathrm{w}}^{2}\right) \sim$ $10^{-2}$, whereas the hadronic branching fractions of the subsequent decay processes are all of the order of one. If these decay channels are kinematically closed, the dominant contribution to hadronic energy release comes from the strongly suppressed four-body decay $\tilde{l} \rightarrow \lambda_{X} l q \bar{q}$, with a branching ratio of the order of $10^{-6}$. Hence, the overall hadronic branching fraction is $B_{\text {had }}^{\tilde{l}} \sim 10^{-6}$ for large masses of the hidden $U(1)$ gaugino $\left(M_{X} \gtrsim M_{\tilde{l}}-M_{Z}\right)$ and $B_{\text {had }}^{\tilde{l}} \sim 10^{-2}$ for small masses $\left(M_{X} \lesssim M_{\tilde{l}}-M_{Z}\right)$. Variations in the corresponding energy release $\epsilon_{\text {had }}^{\tilde{l}}$ are subdominant because $B_{\text {had }}^{\tilde{l}}$ varies by several orders of magnitude, and we simply take $\epsilon_{\text {had }}^{\tilde{l}}=(1 / 3)\left(M_{\tilde{l}}-M_{X}\right)$ 46].

Applying the BBN bounds from Ref. [40] to this scenario, a considerable part of the hidden $U(1)$ gaugino parameter space can be excluded (see Fig. (1) 6 We also show the region that is excluded by the catalytic enhancement of ${ }^{6} \mathrm{Li}$ and ${ }^{9} \mathrm{Be}$ production. These effects essentially require that the lifetime of the slepton is smaller than around $2 \times 10^{3} \mathrm{~s}$ [41].

The impact of superWIMP scenarios on structure formation is encoded in the free streaming length $\lambda_{\mathrm{FS}}$, defined as the comoving distance traveled by a particle since it was produced:

$$
\lambda_{\mathrm{FS}}=\int_{0}^{z_{\mathrm{prod}}} d z \frac{v(z)}{H(z)} .
$$

On scales below the free streaming length of dark matter particles, density fluctuations are washed out before structure formation begins (see e.g. Ref. [4]). The non-observation of this effect in the latest Lyman- $\alpha$ Forest data from the Sloan Digital Sky Survey [4] excludes a free streaming length larger than roughly $\lambda_{\mathrm{FS}} \gtrsim 0.5 \mathrm{Mpc} 7$ The region in the parameter space of the hidden $U(1)$ gaugino that is excluded by this requirement is shown in Fig. 1 and lies completely in the region already ruled out by BBN.

\footnotetext{
${ }^{6}$ In Ref. 40, the bounds are shown for the cases $B_{\text {had }}=1,10^{-3}$ and zero. We just interpolate between $B_{\text {had }}=1$ and $B_{\text {had }}=10^{-3}$ linearly in $\log _{10}\left(B_{\text {had }}\right)$ and use $B_{\text {had }}=0$ as a cutoff.

${ }^{7}$ See Ref. 49, where the power spectrum of non-thermally produced dark matter is confronted with lower bounds on the mass of sterile neutrino dark matter [50, 51].
} 
Scenario ii) Two-body decays of a neutralino NLSP lead to final states where the hidden $U(1)$ gaugino LSP is accompanied either by a $Z^{0}$ or Higgs boson or a photon. However, if the decay into $Z^{0}$ or Higgs bosons is kinematically forbidden, neutralinos predominantly decay via $\lambda_{B} \rightarrow \lambda_{X} f \bar{f}$, since the radiative two-body decay into photons is one-loop suppressed and negligible in most cases. We have summarized all relevant decay widths in appendix $\mathrm{A}$, particularized to the case of a bino-like neutralino. The hadronic branching fraction for the decay of a bino-like neutralino is of the order of one for hidden $U(1)$ gaugino masses that allow the decay into $Z^{0}$ bosons, and can range between $10^{-2}$ and order one otherwise (depending on the squark masses). For definiteness we choose $B_{\text {had }}^{\lambda_{B}} \sim \mathcal{O}(1)$ for all masses $M_{X}$, since smaller values at large $M_{X}$ will not change our conclusions. For the corresponding energy release we simply take $\epsilon_{\text {had }}=1 / 3\left(M_{B}-M_{X}\right)$. We apply the BBN bounds of Ref. [40] and show the corresponding excluded region in Fig. 2. Together with the overproduction bounds, this scenario is excluded in the whole $\left(\chi, M_{X}\right)$ parameter space suggested by string theory, $\chi \sim 10^{-2}-10^{-16}$.

Scenario iii) We will now discuss the effects of an additional light gravitino, concentrating on the spectra shown in Fig. 3, where we also show typical values for the relevant decay widths.

If the hidden $U(1)$ gaugino is the LSP (see Fig. 3a), it can be produced in three different ways: thermally, via slepton decay and via gravitino decay. For mixings and masses that are allowed in Fig. 1 the decay of sleptons into gravitinos is strongly suppressed, and the decay of gravitinos into hidden $U(1)$ gauginos is only accompanied by hidden $U(1)$ gauge bosons and invisible to MSSM particles. Hence, bounds from BBN and overproduction are essentially the same than in case $i$ ).

However, this does not hold for the free streaming bounds, since, in contrast to hidden $U(1)$ gauginos which are produced thermally or via slepton decay, hidden $U(1)$ gauginos that stem from the decay of gravitinos can have a quite large free streaming length, of the order of several Mpc, as shown in Fig. 4. This leads to dark matter with a cold and a warm component, so-called $\Lambda$ CWDM models.

Constraints on $\Lambda$ CWDM models where recently studied in Refs. [52, 53, 54. A warm dark matter component would induce a step in the power spectrum of density fluctuations, with a size which depends on the fraction of the dark matter that is warm and a position which is roughly given by the corresponding free streaming length as $k_{\mathrm{FS}} \sim 2 \pi / \lambda_{\mathrm{FS}}$. Although models 
with mixed cold/warm dark matter arise quite naturally in many situations, a full general analysis with all the latest data is still lacking 8 Bearing in mind this limitation, we will simply show the corresponding bounds on the reheating temperature and mixing parameter for the two reference values $f=0.2$ and $f=0.02$, where $f$ denotes the fraction of dark matter that is warm, $\Omega_{\mathrm{DM}}=f \Omega_{\mathrm{WDM}}+(1-f) \Omega_{\mathrm{CDM}}$; a complete analysis of the mixed cold/warm dark matter scenario is beyond the scope of this paper.

As discussed above, the warm component of hidden $U(1)$ gaugino DM comes from the late decay of gravitinos. The thermal abundance of gravitinos directly depends on the reheating temperature $T_{R}$ like [36, 37, 38]

$$
\Omega_{\tilde{G}} h^{2} \simeq 0.27\left(\frac{T_{R}}{10^{10} \mathrm{GeV}}\right)\left(\frac{100 \mathrm{GeV}}{m_{\tilde{G}}}\right)\left(\frac{m_{\tilde{g}}}{1 \mathrm{TeV}}\right)^{2}
$$

where $m_{\tilde{g}}$ denotes the gluino mass. Hence, the bounds on $\Lambda$ CWDM models translate into a bound on the reheating temperature as shown in the right part of Fig. 5.

Scenario iv) Scenarios with a gravitino LSP and a slepton (usually a stau) NLSP are known to be strongly constrained due to the catalytic production of ${ }^{6} \mathrm{Li}$ and ${ }^{9} \mathrm{Be}$ in the presence of long-lived charged particles during $\mathrm{BBN}$ (see above discussion). The resulting bound on the lifetime of staus translates into an upper bound on the gravitino mass of around $m_{\tilde{G}} \lesssim 1 \mathrm{GeV}$. At the same time, requiring that gravitinos with $m_{\tilde{G}} \lesssim 1 \mathrm{GeV}$ are not overproduced forbids reheating temperatures as high as $T_{R} \sim 10^{9} \mathrm{GeV}$ (see left part of Fig. 5 and Eq. (18) ). However, these high reheating temperatures are favored in scenarios with leptogenesis as the source of the baryon asymmetry of the universe [56, 57].

One way to relax the tension between leptogenesis and gravitino DM is to assume that the production of entropy between stau freeze out and BBN dilutes the stau abundance sufficiently to evade the bounds [58, 59].

\footnotetext{
${ }^{8}$ In Ref. [54] a consideration of the uncertainty of the power spectrum from WMAP 1 -yr data [55] bounds the fraction $f$ of dark matter that is allowed to have $\lambda_{\mathrm{FS}} \simeq 6 \mathrm{Mpc}$ to be $f \lesssim 0.2-0.4$. This seems to be consistent with a hydrodynamical analysis in Ref. [52, where the bound on the fraction of dark matter that can be made out of thermal light gravitinos with a mass of $m_{\tilde{G}} \approx 16 \mathrm{eV}$ is stated as $f \lesssim 0.12$, since the corresponding free streaming length (using Eq. (17)) is around $\lambda_{\mathrm{FS}} \sim 40 \mathrm{Mpc}$. However, both analyses do not take into account the latest Lyman- $\alpha$-forest data [48. An analysis in Ref. [53] which incorporates these data, and where lower bounds on the mass of sterile neutrino dark matter from Ref. 50 are rescaled for the case of $\Lambda$ CWDM models, suggests that even a component with a free streaming lengths around $\lambda_{\mathrm{FS}} \sim 4 \mathrm{Mpc}$ is bounded strongly with $f \lesssim 0.1$.
} 
Another way is to impose a small violation of $R$-parity [6], which allows the stau to decay fast into standard model particles. In this case, the gravitino becomes unstable and decays with a lifetime which is typically longer than the age of the universe. The gravitino decay products could be detected as an anomalous contribution to the cosmic ray fluxes [60, 61, 62, opening the possibility to probe this scenario.

Interestingly, a similar mechanism can work in the presence of a hidden $U(1)$ gaugino for spectra of the form (slepton, $\lambda_{X}$, gravitino), as depicted in Fig. 3b. For mixings and masses of the hidden $U(1)$ gaugino that lie in the allowed region of Fig. 1, the lifetime of the stau is compatible with all $\mathrm{BBN}$ bounds. In this case, the reheating temperature can be as large as $T_{R} \sim 10^{9} \mathrm{GeV}$, as shown in Fig. 5, since the gravitino mass and the stau lifetime are decoupled.

The warm component of gravitino dark matter, with a free streaming length of the order of several Mpc (see Fig. 4), comes now from the late decay of hidden $U(1)$ gauginos. Hence, bounds on $\Lambda$ CWDM models constrain the sum over the thermal and non-thermal abundance of hidden $U(1)$ gauginos in this case. Firstly, this shrinks the allowed region of the hidden $U(1)$ gaugino parameter space as shown in Fig. 1(dashed lines). Secondly, in cases where the thermal production of hidden $U(1)$ gauginos is negligible, the stau abundance is bounded like $Y_{\text {stau }} \lesssim\left(9 \times 10^{-14}-9 \times 10^{-13}\right)\left(M_{\tilde{G}} / 100 \mathrm{GeV}\right)^{-1}$ if we allow a fraction $f=0.02-0.2$ of dark matter to be warm. Thus, the ${ }^{9} \mathrm{Be}$ and ${ }^{6} \mathrm{Li}$ bounds on the abundance of long-lived staus, which are roughly

given by $Y_{\text {stau }} \lesssim 10^{-15}$ and $Y_{\text {stau }} \lesssim 10^{-16}$, respectively (see Ref. [44]), can be weakened by two or three orders of magnitude.

In the CMSSM with gravitino dark matter the typical relic abundance of stau NLSPs ranges between $Y_{\text {stau }} \sim 10^{-14}$ and $Y_{\text {stau }} \sim 10^{-13}$ (although considerably smaller values are possible due to resonant effects or coannihilation [63], or e.g. in models with non-universal Higgs masses [64]). In these kind of models, the phenomenologically allowed parameter space will be greatly enlarged by the above mechanism. However, a detailed analysis of the influence of a weakly interacting hidden sector on constraints on the reheating temperature would require reliable bounds for general $\Lambda \mathrm{CWDM}$ models.

\section{Collider phenomenology}

At the LHC, cascade decays of squarks and gluinos can produce of the order of $10^{6}$ NLSPs per year if the sparticle masses are close to the current 
experimental limits 65, thus offering a unique opportunity to test scenarios with a light hidden $U(1)$ gaugino. It was shown in the previous sections that in this class of scenarios the neutralino as NLSP is excluded, whereas the stau as NLSP is allowed only if it has a lifetime that ranges between $\tau \sim 10^{-2} \mathrm{~s}$ and $\tau \sim 10^{3} \mathrm{~s}$. Therefore, the stau will typically traverse the detector leaving a distinctive heavily ionizing charged track [66. Moreover, if the stau is slow enough, it might get trapped in the detector, decaying at late times and thus allowing a measurement of the lifetime and, correspondingly, of the mixing parameter $\chi$. The number of staus trapped can be incremented by placing a 1-10 kton massive material around the LHC detectors, which will allow to collect $\mathcal{O}\left(10^{3}-10^{4}\right)$ staus 67. If the stopper material is simultaneously an active real-time detector, the decay products and their angular distribution could be studied in detail. This will allow to distinguish this scenario from the scenario of gravitino dark matter, which yields very similar signatures at colliders 68 .

\section{The case with an anomalously small $\chi$}

We have shown in sections 3 and 4 that constraints from overproduction, BBN and structure formation exclude the scenario with a neutralino NLSP and hidden $U(1)$ gaugino for natural values of the mixing parameter $\chi \sim$ $10^{-2}-10^{-16}$ (see Fig. 2 ). However, these strong constraints could be evaded in more elaborated models yielding a smaller $\chi$. Our interest in exploring the region with small $\chi$ is that the neutralino lifetime could become larger than the age of the Universe, thus constituting a viable dark matter candidate itself 9 Nevertheless, in contrast to the standard neutralino dark matter scenario, in this scenario the neutralino NLSP is unstable and decays with very long lifetimes into the hidden gaugino and Standard Model particles.

The neutralino dark matter particle (that for definiteness we assume to be a pure bino) can decay into different channels:

$$
\begin{aligned}
& \lambda_{B} \rightarrow Z \lambda_{X}, \\
& \lambda_{B} \rightarrow \gamma \lambda_{X}, \\
& \lambda_{B} \rightarrow h \lambda_{X}, \\
& \lambda_{B} \rightarrow f \bar{f} \lambda_{X},
\end{aligned}
$$

\footnotetext{
${ }^{9}$ Clearly, the scenario with charged slepton NLSP is excluded for very small values of $\chi$, since it would yield an abundance of anomalously heavy hydrogen in conflict with the experimental constraints.
} 
with decay rates listed in appendix $\mathrm{A}$. The fragmentation of the $Z^{0}$ and the Higgs boson produces a continuous spectrum of stable particles, such as photons, positrons or antiprotons which contribute to the primary fluxes of cosmic rays. The experimental constraints on these fluxes will then translate into constraints on the parameters of the model.

On the other hand, it has been pointed out recently that the decay of a dark matter particle with a lifetime $\tau \sim 10^{26}$ s into weak gauge bosons with a momentum $\sim 50 \mathrm{GeV}$ could simultaneously explain the EGRET anomaly in the extragalactic gamma-ray background and the HEAT excess in the positron fraction [61, 62]. Interestingly, the scenario we are considering here, where the neutralino dark matter particle decays into a $Z^{0}$ gauge boson, constitutes a promising candidate of this kind. Moreover, in contrast to other candidates for decaying dark matter that have been proposed, such as the gravitino [6] or a hidden $U(1)$ gauge boson [69], the unstable neutralino could be detected in direct dark matter searches.

Although the simplest models with hidden gaugino LSP and neutralino NLSP predict a mixing parameter $\chi$ ranging between $10^{-2}$ and $10^{-16}$, and thus a neutralino lifetime ranging between $\tau \sim 10^{-14} \mathrm{~s}$ and $\tau \sim 10^{14} \mathrm{~s}$, it is not difficult to construct plausible models where the neutralino lifetime can be orders of magnitude longer than the age of the Universe. To this end, let us consider a model with two hidden $U(1)$ gauge groups, $U(1)_{X}$ and $U(1)_{X^{\prime}}$, and vector superfields denoted by $\hat{X}$ and $\hat{X}^{\prime}$, respectively. We will assume that the hidden sector particles that generate the kinetic mixing are charged either under $U(1)_{Y}$ and $U(1)_{X^{\prime}}$, or under $U(1)_{X}$ and $U(1)_{X^{\prime}}$, but not under $U(1)_{Y}$ and $U(1)_{X}$ simultaneously. If this is the case, the kinetic mixings $\hat{W}_{B}^{\alpha} \hat{W}_{X^{\prime} \alpha}$ and $\hat{W}_{X}^{\alpha} \hat{W}_{X^{\prime} \alpha}$ will be generated, but not $\hat{W}_{B}^{\alpha} \hat{W}_{X \alpha}$. Therefore, the SUSY invariant part of the Lagrangian reads

$$
\begin{aligned}
\mathcal{L}_{\mathrm{SUSY}}= & \int d^{2} \theta\left(\hat{W}_{B}^{\alpha} \hat{W}_{B \alpha}+\hat{W}_{X}^{\alpha} \hat{W}_{X \alpha}+\hat{W}_{X^{\prime}}^{\alpha} \hat{W}_{X^{\prime} \alpha}+\right. \\
& \left.2 \chi_{1} \hat{W}_{B}^{\alpha} \hat{W}_{X^{\prime} \alpha}+2 \chi_{2} \hat{W}_{X}^{\alpha} \hat{W}_{X^{\prime} \alpha}\right)+ \text { h.c. }
\end{aligned}
$$

In addition, there exists a soft SUSY breaking Lagrangian:

$$
\mathcal{L}_{\text {SUSY }}=-\frac{1}{2}\left(\begin{array}{lll}
\hat{\lambda}_{X} & \hat{\lambda}_{X^{\prime}} & \hat{\lambda}_{B}
\end{array}\right) \hat{\mathcal{M}}\left(\begin{array}{c}
\hat{\lambda}_{X} \\
\hat{\lambda}_{X^{\prime}} \\
\hat{\lambda}_{B}
\end{array}\right)+\text { h.c. },
$$

where

$$
\hat{\mathcal{M}}=\left(\begin{array}{ccc}
\hat{M}_{X} & \delta \hat{M}_{2} & 0 \\
\delta \hat{M}_{2} & \hat{M}_{X^{\prime}} & \delta \hat{M}_{1} \\
0 & \delta \hat{M}_{1} & \hat{M}_{B}
\end{array}\right)
$$


This structure for the soft mass matrix can be straightforwardly derived from Eq. (20) introducing five arbitrary spurion superfields as in Eq. (9). Note that $\delta \hat{M}_{1}$ and $\delta \hat{M}_{2}$ are suppressed by $\chi_{1}$ and $\chi_{2}$, respectively.

In order to canonically normalize the vector superfields, we define

$$
\begin{aligned}
B & =\hat{B} \\
X & =\hat{X}-\chi_{1} \chi_{2} \hat{B}, \\
X^{\prime} & =\hat{X}^{\prime}+\chi_{1} \hat{B}+\chi_{2} \hat{X}
\end{aligned}
$$

where we have assumed $\chi_{1}, \chi_{2} \ll 1$. Then, the SUSY invariant part of the Lagrangian reads:

$$
\mathcal{L}_{\mathrm{SUSY}}=\int d^{2} \theta\left(W_{B}^{\alpha} W_{B \alpha}+W_{X}^{\alpha} W_{X \alpha}+W_{X^{\prime}}^{\alpha} W_{X^{\prime} \alpha}\right)+\text { h.c. },
$$

while the SUSY breaking Lagrangian reads:

$$
\mathcal{L}_{\text {SUSY }}=-\frac{1}{2}\left(\begin{array}{lll}
\lambda_{X} & \lambda_{X^{\prime}} & \lambda_{B}
\end{array}\right) \mathcal{M}\left(\begin{array}{c}
\lambda_{X} \\
\lambda_{X^{\prime}} \\
\lambda_{B}
\end{array}\right)+\text { h.c. },
$$

with

$$
\mathcal{M} \simeq\left(\begin{array}{ccc}
M_{X} & \delta M_{2} & \delta M_{12} \\
\delta M_{2} & M_{X^{\prime}} & \delta M_{1} \\
\delta M_{12} & \delta M_{1} & M_{B}
\end{array}\right)
$$

In this equation, $M_{X} \simeq \hat{M}_{X}, M_{X^{\prime}} \simeq \hat{M}_{X^{\prime}}$ and $M_{B} \simeq \hat{M}_{B}$, while $\delta M_{2} \simeq$ $\delta \hat{M}_{2}-\chi_{2} M_{X^{\prime}}$ and $\delta M_{1} \simeq \delta \hat{M}_{1}-\chi_{1} M_{X}$. More importantly, after the canonical normalization of the kinetic terms, a mixing term between the bino and the hidden gaugino $\lambda_{X}$ has been generated, $\delta M_{12} \simeq \chi_{1} \chi_{2}\left(M_{X}+\right.$ $\left.M_{X^{\prime}}\right)-\chi_{2} \delta \hat{M}_{1}-\chi_{1} \delta \hat{M}_{2}$, which is doubly suppressed by $\chi_{1}$ and $\chi_{2}$.

If $\lambda_{X}$ is the LSP and $\lambda_{B}$ is the NLSP, the decay rate of the bino NLSP is very strongly suppressed, thus yielding very long lifetimes for the neutralino dark matter. For instance, assuming that the kinetic mixing between the observable sector and the hidden sector is $\chi_{1} \sim 10^{-16}$, as suggested by string theory, a mixing between the two hidden sector $U(1)$ s of $\chi_{2} \sim 10^{-7}$ would be necessary in order to render a neutralino lifetime of the order of $10^{26} \mathrm{~s}$.

The signatures in cosmic ray experiments of the scenario of neutralino dark matter which decays into hidden gauginos are fairly model dependent and will be shown elsewhere [70. In this paper we will just derive a conservative bound on the mixing parameter $\chi$ from requiring a gamma ray flux from neutralino decay in agreement with the EGRET observations. To this 
end, we will just consider the contribution to the gamma ray flux from the two body decay $\lambda_{B} \rightarrow \gamma \lambda_{X}$.

The total gamma ray flux receives several contributions. First, we expect a gamma ray flux from the center of the Galaxy produced by neutralino annihilations. This contribution does not depend on whether the neutralino is absolutely stable or on the contrary decays at late times. Hence, it does not constrain our scenario and will not be further discussed. The interested reader can find in the vast existing literature the prospects of detecting such a signal from neutralino annihilation coming from the center of the Galaxy (see e.g. Ref. [1]).

In addition, we expect a diffuse flux produced in the decay of neutralinos at cosmological distances and in the Milky Way halo. Following Ref. [6, 71, we will average the halo gamma ray signal of the $\lambda_{B} \rightarrow \gamma \lambda_{X}$ channel over the whole sky, excluding a band of $\pm 10^{\circ}$ around the Galactic disk in order to compare our results with the EGRET data [72]. To be conservative, we will require that the corresponding peak in the energy spectrum, after convolving with the energy resolution of $15 \%$ quoted by EGRET, remains below the $2 \sigma$ band of the EGRET spectrum obtained by Sreekumar et al. in [73],

$\frac{d J_{\mathrm{EGRET}}}{d E}=(7.32 \pm 0.34) \times 10^{-6}\left(\mathrm{~cm}^{2} \mathrm{~s} \mathrm{str} \mathrm{GeV}\right)^{-1}\left(\frac{E}{0.451 \mathrm{GeV}}\right)^{-2.1 \pm 0.03}$

The result is shown in Fig. 6, where we used a reference bino mass of $M_{B}=$ $150 \mathrm{GeV}$. For hidden $U(1)$ gaugino masses around $M_{X} \sim 75 \mathrm{GeV}$, mixings down to $\chi \sim 10^{-21}$ can be excluded from the EGRET measurements of the gamma ray flux.

\section{Conclusions}

An unbroken hidden $U(1)$ that interacts with the Standard Model only via kinetic mixing with hypercharge decouples completely from the visible world. However, in the supersymmetric version of this scenario this is no longer the case. We have shown that a mass mixing between the bino and the hidden $U(1)$ gaugino is always generated via radiative effects, although this mixing can be generated already at tree level in some well motivated scenarios. Moreover, we have discussed different scenarios of supersymmetry breaking in which the hidden $U(1)$ gaugino is the lightest supersymmetric particle.

We have mostly concentrated on this possibility and we have derived cosmological bounds on this scenario from precluding overproduction of hidden 
$U(1)$ gauginos and from the requirements of successful Big Bang nucleosynthesis and structure formation. The combination of these constraints excludes a neutralino NLSP except for extremely small mixings (see Fig. 2). On the other hand, when the NLSP is a stau, an allowed window for mixings around $\chi \sim 10^{-10}-10^{-13}$ remains (see Fig. 1). In this window, the stau has a lifetime larger than $10^{-1} \mathrm{~s}$ and thus might be detected at future colliders as a heavily ionizing charged track.

The reheating temperature in scenarios with gravitino dark matter and stau NLSPs is known to be strongly constrained, and we have shown that these constraints relax considerably in the presence of a hidden $U(1)$ gaugino (see Fig. 5). This might be a rather general effect of very weakly interacting hidden sectors and deserves further attention.

Finally, we have discussed the case of an anomalously small mixing parameter, $\chi \ll 10^{-16}$. For these small mixings, the neutralino NLSP can become long lived enough to constitute the dark matter of the Universe. We have constructed a simple model with two hidden $U(1)$ s where a tiny mixing can be naturally obtained. Even though the neutralino is very long lived, it eventually decays into the hidden gaugino and standard model particles, which might be detected as an anomalous contribution to the cosmic ray fluxes. Using the EGRET measurement of the extragalactic gamma ray flux, we have derived a conservative bound on the mixing parameter $\chi \lesssim 10^{-20-21}$ (see Fig. 6).

\section{Acknowledgments}

C.W. thanks Chloé Papineau, James Wells, and especially Javier Redondo for valuable discussions.

\section{A Decay rates}

For convenience, we summarize the neutralino, slepton and gravitino decay rates that we used in this paper.

The decay widths for bino-like neutralinos that decay into hidden $U(1)$ 
gauginos can be derived from the results in Ref. [74, 75]. They are

$$
\begin{aligned}
& \Gamma\left(\lambda_{B} \rightarrow Z^{0} \lambda_{X}\right) \simeq \frac{1}{128 \pi} g^{\prime 2} s_{W}^{2} \Theta^{2} M_{B} \lambda^{1 / 2}\left(1, \frac{M_{X}^{2}}{M_{B}^{2}}, \frac{M_{Z}^{2}}{M_{B}^{2}}\right) \times \\
& \times \frac{M_{Z}^{6}}{\mu^{4} M_{B}^{2}}\left(\frac{M_{B}^{2}}{M_{Z}^{2}}+\frac{M_{X}^{2}}{M_{Z}^{2}}-2+\left(\frac{M_{B}^{2}}{M_{Z}^{2}}-\frac{M_{X}^{2}}{M_{Z}^{2}}\right)^{2}+6 \frac{M_{X}}{M_{Z}} \frac{M_{B}}{M_{Z}}\right), \\
& \Gamma\left(\lambda_{B} \rightarrow \gamma \lambda_{X}\right) \simeq \frac{1}{128 \pi}\left(\frac{15}{32 \pi^{2}}\right)^{2} e^{2} g^{\prime 4} \Theta^{2} M_{B} \times \\
& \times \frac{M_{B}^{4}}{M_{\mathrm{sf}}^{4}}\left(1-\frac{M_{X}^{2}}{M_{B}^{2}}\right)^{3}\left(1-\frac{M_{X}}{M_{B}}\right)^{2}, \\
& \Gamma\left(\lambda_{B} \rightarrow h \lambda_{X}\right) \simeq \frac{1}{32 \pi} g^{\prime 2} \Theta^{2} M_{B} \frac{M_{Z}^{2} s_{W}^{2}}{\mu^{2}} \lambda^{1 / 2}\left(1, \frac{M_{X}^{2}}{M_{B}^{2}}, \frac{M_{h}^{2}}{M_{B}^{2}}\right) \times \\
& \times\left(1+\frac{M_{X}^{2}}{M_{B}^{2}}-\frac{M_{h}^{2}}{M_{B}^{2}}+2 \frac{M_{X}}{M_{B}}\right), \\
& \Gamma\left(\lambda_{B} \rightarrow f \bar{f} \lambda_{X}\right) \simeq 1.4 \times 10^{-4} g^{\prime 4} \Theta^{2} M_{B} \frac{M_{B}^{4}}{M_{\mathrm{sf}}^{4}}\left(1-\frac{M_{X}^{2}}{M_{B}^{2}}\right)^{5},
\end{aligned}
$$

where we have used the function

$$
\lambda\left(a^{2}, b^{2}, c^{2}\right)=\left(a^{2}-(b+c)^{2}\right)\left(a^{2}-(b-c)^{2}\right) .
$$

The approximations that we use for the neutralino mixing angles become exact in the limit $M_{X} \ll M_{B} \ll M_{W} \ll \mu$. Note that the two-body decay into a hidden $U(1)$ gaugino and a photon, Eq. (29), is one-loop suppressed, and we sum over all (s)leptons in the loop, assuming that they have a common mass $M_{\mathrm{sf}}$. The final state in Eq. (31) incorporates neutrinos and charged leptons, and we took into account sfermions and $Z^{0}$ gauge bosons in the intermediate state, where the later are subdominant.

The relevant decay widths with gravitinos in the initial or final state can be found in Ref. [46, 76] and are

$$
\begin{aligned}
\Gamma\left(\lambda_{X} \rightarrow \tilde{G} X\right) & =\frac{1}{48 \pi M_{\mathrm{pl}}^{2}} \frac{M_{X}^{5}}{M_{\tilde{G}}^{2}}\left(1-\frac{M_{\tilde{G}}^{2}}{M_{X}^{2}}\right)^{3}\left(1+3 \frac{M_{\tilde{G}}^{2}}{M_{X}^{2}}\right), \\
\Gamma\left(\tilde{G} \rightarrow \lambda_{X} X\right) & =\frac{1}{32 \pi M_{\mathrm{pl}}^{2}} M_{\tilde{G}}^{3}\left(1-\frac{M_{X}^{2}}{M_{\tilde{G}}^{2}}\right)^{3}\left(1+\frac{1}{3} \frac{M_{X}^{2}}{M_{\tilde{G}}^{2}}\right), \\
\Gamma(\tilde{l} \rightarrow \tilde{G} l) & =\frac{1}{48 \pi M_{\mathrm{pl}}^{2}} \frac{M_{\tilde{l}}^{5}}{M_{\tilde{G}}^{2}}\left(1-\frac{M_{\tilde{G}}^{2}}{M_{\tilde{l}}^{2}}\right)^{4} .
\end{aligned}
$$




\section{B Renormalization group equations}

The RGEs for multiple $U(1)$ models with kinetic mixing where first published in Ref. [77. One-loop RGEs for gaugino masses and their mixings can be found in Ref. [78, 10

The RGEs acquire their simplest form in the basis where gauge bosons and gauginos have a canonical kinetic term. The matrix of the gauge couplings $\bar{g}_{i j}$ is defined according to the term $\mathcal{L} \supset \bar{g}_{i j} j_{i}^{\mu} A_{\mu}^{j}$ in the Lagrangian, where the indices $i=1,2$ and $j=1,2$ run over the charged currents of the two sectors and over the gauge boson states, respectively. In this representation, the one-loop RGEs for the coupling constants are

$$
\frac{d}{d t} \bar{g}=\frac{1}{16 \pi^{2}} \bar{g} \bar{g}^{T} B \bar{g}
$$

where we have used the charge matrix $B_{i j}=\operatorname{tr}\left(Q_{i} Q_{j}\right)$, and $t=\ln \left(Q / Q_{0}\right)$ with $Q$ as RG scale. The trace in $B_{i j}$ runs over all chiral supermultiplets.

The RGEs for the gaugino mass matrix $\overline{\mathcal{M}}$ take a similar form

$$
\frac{d}{d t} \overline{\mathcal{M}}=\frac{1}{16 \pi^{2}}\left(\overline{\mathcal{M}} \bar{g}^{T} B \bar{g}+\bar{g}^{T} B \bar{g} \overline{\mathcal{M}}\right)
$$

Note that only three of the four entries of $\bar{g}$ are physical because the basis of the gauge bosons is only fixed up to a rotation. It is convenient to state the RGEs for the case where the non-diagonal elements in the couplings $g_{i j}$ are chosen to vanish, and where their third free component is absorbed in the kinetic mixing term like in Eq. (5). We obtain

$$
\begin{gathered}
\frac{d}{d t} g_{X, B}=\frac{1}{16 \pi^{2}} g_{X, B}^{3} B_{X X, B B}, \\
\frac{d}{d t} \chi=-\frac{1}{8 \pi^{2}} g_{X} g_{B} B_{X B}+\frac{1}{16 \pi^{2}} \chi\left(g_{X}^{2} B_{X X}+g_{B}^{2} B_{B B}\right)+\mathcal{O}\left(\chi^{2}\right), \\
\frac{d}{d t} \hat{M}_{X, B}=\frac{1}{8 \pi^{2}} g_{X, B}^{2} B_{X X, B B} \hat{M}_{X, B}, \\
\frac{d}{d t} \delta \hat{M}=\frac{1}{16 \pi^{2}}\left(g_{X}^{2} B_{X X}+g_{B}^{2} B_{B B}\right) \delta \hat{M}+\mathcal{O}\left(\chi^{2}\right) .
\end{gathered}
$$

\footnotetext{
${ }^{10}$ Note that our result for the RGEs of the gaugino mass matrix in Eq. (36) differs from the one given in Ref. [78 by an additional symmetrization.
} 


\section{References}

[1] G. Bertone, D. Hooper, and J. Silk, Phys. Rept. 405, 279 (2005), hep$\mathrm{ph} / 0404175$.

[2] J. R. Ellis, J. S. Hagelin, D. V. Nanopoulos, K. A. Olive, and M. Srednicki, Nucl. Phys. B238, 453 (1984).

[3] G. Jungman, M. Kamionkowski, and K. Griest, Phys. Rept. 267, 195 (1996), hep-ph/9506380.

[4] H. Pagels and J. R. Primack, Phys. Rev. Lett. 48, 223 (1982).

[5] F. Takayama and M. Yamaguchi, Phys. Lett. B485, 388 (2000), hep$\mathrm{ph} / 0005214$.

[6] W. Buchmuller, L. Covi, K. Hamaguchi, A. Ibarra, and T. Yanagida, JHEP 03, 037 (2007), hep-ph/0702184.

[7] B. Holdom, Phys. Lett. B166, 196 (1986).

[8] R. Foot and X.-G. He, Phys. Lett. B267, 509 (1991).

[9] L. B. Okun, Sov. Phys. JETP 56, 502 (1982).

[10] B. Holdom, Phys. Lett. B259, 329 (1991).

[11] K. R. Dienes, C. F. Kolda, and J. March-Russell, Nucl. Phys. B492, 104 (1997), hep-ph/9610479.

[12] R. Blumenhagen, S. Moster, and T. Weigand, Nucl. Phys. B751, 186 (2006), hep-th/0603015.

[13] D. Lüst and S. Stieberger, Fortsch. Phys. 55, 427 (2007), hepth/0302221.

[14] S. A. Abel and B. W. Schofield, Nucl. Phys. B685, 150 (2004), hepth/0311051.

[15] M. Berg, M. Haack, and B. Kors, Phys. Rev. D71, 026005 (2005), hep-th/0404087.

[16] S. A. Abel, J. Jaeckel, V. V. Khoze, and A. Ringwald, Phys. Lett. B666, 66 (2008), hep-ph/0608248. 
[17] S. A. Abel, M. D. Goodsell, J. Jaeckel, V. V. Khoze, and A. Ringwald, JHEP 07, 124 (2008), 0803.1449.

[18] S. Davidson and M. E. Peskin, Phys. Rev. D49, 2114 (1994), hep$\mathrm{ph} / 9310288$.

[19] S. Davidson, S. Hannestad, and G. Raffelt, JHEP 05, 003 (2000), hep$\mathrm{ph} / 0001179$.

[20] A. Melchiorri, A. Polosa, and A. Strumia, Phys. Lett. B650, 416 (2007), hep-ph/0703144.

[21] W.-F. Chang, J. N. Ng, and J. M. S. Wu, Phys. Rev. D74, 095005 (2006), hep-ph/0608068.

[22] P. Langacker, (2008), 0801.1345.

[23] D. Feldman, B. Kors, and P. Nath, Phys. Rev. D75, 023503 (2007), hep-ph/0610133.

[24] D. Feldman, Z. Liu, and P. Nath, Phys. Rev. D75, 115001 (2007), hep-ph/0702123.

[25] B. Kors and P. Nath, JHEP 07, 069 (2005), hep-ph/0503208.

[26] J. Kumar and J. D. Wells, Phys. Rev. D74, 115017 (2006), hep$\mathrm{ph} / 0606183$.

[27] B. Kors and P. Nath, JHEP 12, 005 (2004), hep-ph/0406167.

[28] K. Cheung and T.-C. Yuan, JHEP 03, 120 (2007), hep-ph/0701107.

[29] M. Pospelov, A. Ritz, and M. B. Voloshin, (2008), 0807.3279.

[30] B. A. Dobrescu, Phys. Rev. Lett. 94, 151802 (2005), hep-ph/0411004.

[31] E. Boos, V. Bunichev, and H. J. Schreiber, Phys. Rev. D78, 015007 (2008), 0709.4535.

[32] J. L. Feng, H. Tu, and H.-B. Yu, (2008), 0808.2318.

[33] J. Redondo, (2008), 0805.3112.

[34] J. Redondo, JCAP 0807, 008 (2008), 0801.1527.

[35] J. Jaeckel, J. Redondo, and A. Ringwald, (2008), 0804.4157. 
[36] M. Bolz, A. Brandenburg, and W. Buchmuller, Nucl. Phys. B606, 518 (2001), hep-ph/0012052, [Erratum-ibid. B 790 (2008) 336].

[37] J. Pradler and F. D. Steffen, Phys. Rev. D75, 023509 (2007), hep$\mathrm{ph} / 0608344$.

[38] V. S. Rychkov and A. Strumia, Phys. Rev. D75, 075011 (2007), hep$\mathrm{ph} / 0701104$.

[39] E. Braaten and T. C. Yuan, Phys. Rev. Lett. 66, 2183 (1991).

[40] M. Kawasaki, K. Kohri, and T. Moroi, Phys. Rev. D71, 083502 (2005), astro-ph/0408426.

[41] M. Pospelov, Phys. Rev. Lett. 98, 231301 (2007), hep-ph/0605215.

[42] J. L. Feng, A. Rajaraman, and F. Takayama, Phys. Rev. Lett. 91, 011302 (2003), hep-ph/0302215.

[43] K. Hamaguchi, T. Hatsuda, M. Kamimura, Y. Kino, and T. T. Yanagida, Phys. Lett. B650, 268 (2007), hep-ph/0702274.

[44] M. Pospelov, J. Pradler, and F. D. Steffen, (2008), 0807.4287.

[45] J. A. R. Cembranos, J. L. Feng, A. Rajaraman, and F. Takayama, Phys. Rev. Lett. 95, 181301 (2005), hep-ph/0507150.

[46] J. L. Feng, S. Su, and F. Takayama, Phys. Rev. D70, 075019 (2004), hep-ph/0404231.

[47] E. W. Kolb and M. S. Turner, The Early Universe (Front. Phys., 1990).

[48] SDSS, P. McDonald et al., Astrophys. J. Suppl. 163, 80 (2006), astro$\mathrm{ph} / 0405013$.

[49] L. E. Strigari, M. Kaplinghat, and J. S. Bullock, Phys. Rev. D75, 061303 (2007), astro-ph/0606281.

[50] U. Seljak, A. Makarov, P. McDonald, and H. Trac, Phys. Rev. Lett. 97, 191303 (2006), astro-ph/0602430.

[51] M. Viel, J. Lesgourgues, M. G. Haehnelt, S. Matarrese, and A. Riotto, Phys. Rev. Lett. 97, 071301 (2006), astro-ph/0605706.

[52] M. Viel, J. Lesgourgues, M. G. Haehnelt, S. Matarrese, and A. Riotto, Phys. Rev. D71, 063534 (2005), astro-ph/0501562. 
[53] A. Palazzo, D. Cumberbatch, A. Slosar, and J. Silk, Phys. Rev. D76, 103511 (2007), 0707.1495.

[54] K. Ishiwata, S. Matsumoto, and T. Moroi, Phys. Rev. D77, 035004 (2008), 0710.2968.

[55] WMAP, D. N. Spergel et al., Astrophys. J. Suppl. 148, 175 (2003), astro-ph/0302209.

[56] M. Fukugita and T. Yanagida, Phys. Lett. B174, 45 (1986).

[57] W. Buchmuller, P. Di Bari, and M. Plumacher, Ann. Phys. 315, 305 (2005), hep-ph/0401240.

[58] W. Buchmuller, K. Hamaguchi, M. Ibe, and T. T. Yanagida, Phys. Lett. B643, 124 (2006), hep-ph/0605164.

[59] J. Pradler and F. D. Steffen, Phys. Lett. B648, 224 (2007), hep$\mathrm{ph} / 0612291$.

[60] A. Ibarra and D. Tran, Phys. Rev. Lett. 100, 061301 (2008), 0709.4593.

[61] A. Ibarra and D. Tran, JCAP 0807, 002 (2008), 0804.4596.

[62] K. Ishiwata, S. Matsumoto, and T. Moroi, (2008), 0805.1133.

[63] J. Pradler and F. D. Steffen, (2008), 0808.2462.

[64] M. Ratz, K. Schmidt-Hoberg, and M. W. Winkler, (2008), 0808.0829.

[65] W. Beenakker, R. Hopker, M. Spira, and P. M. Zerwas, Nucl. Phys. B492, 51 (1997), hep-ph/9610490.

[66] M. Drees and X. Tata, Phys. Lett. B252, 695 (1990).

[67] K. Hamaguchi, Y. Kuno, T. Nakaya, and M. M. Nojiri, Phys. Rev. D70, 115007 (2004), hep-ph/0409248.

[68] W. Buchmuller, K. Hamaguchi, M. Ratz, and T. Yanagida, Phys. Lett. B588, 90 (2004), hep-ph/0402179.

[69] C.-R. Chen, F. Takahashi, and T. T. Yanagida, (2008), 0809.0792.

[70] In preparation.

[71] G. Bertone, W. Buchmuller, L. Covi, and A. Ibarra, JCAP 0711, 003 (2007), 0709.2299. 
[72] A. W. Strong, I. V. Moskalenko, and O. Reimer, Astrophys. J. 613, 956 (2004), astro-ph/0405441.

[73] EGRET, P. Sreekumar et al., Astrophys. J. 494, 523 (1998), astro$\mathrm{ph} / 9709257$.

[74] H. E. Haber and D. Wyler, Nucl. Phys. B323, 267 (1989).

[75] A. Bartl, H. Fraas, and W. Majerotto, Nucl. Phys. B278, 1 (1986).

[76] K. Kohri, T. Moroi, and A. Yotsuyanagi, Phys. Rev. D73, 123511 (2006), hep-ph/0507245.

[77] F. del Aguila, G. D. Coughlan, and M. Quiros, Nucl. Phys. B307, 633 (1988).

[78] D. Suematsu, Phys. Rev. D59, 055017 (1999), hep-ph/9808409. 


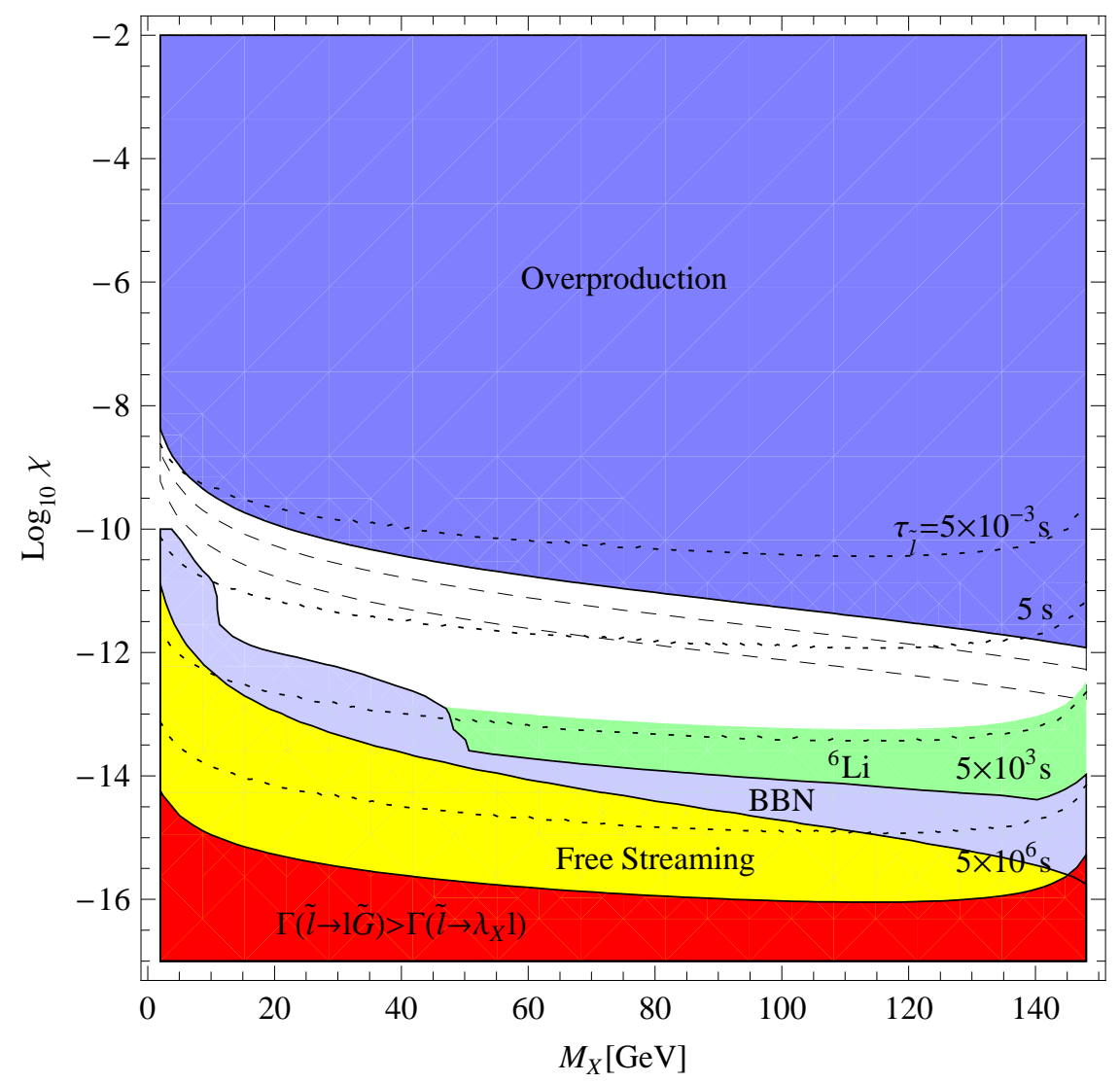

Figure 1: Summary of bounds on the hidden $U(1)$ gaugino parameter space for the case of a slepton NLSP. We use $M_{\tilde{l}}=150 \mathrm{GeV}$ and $M_{B}=180 \mathrm{GeV}$. The upper dark blue region is excluded by thermal overproduction. Below this region, the hidden $U(1)$ gaugino is dominantly produced via late decaying sleptons. The light blue region is excluded by energy injection during $\mathrm{BBN}$ [40], whereas the light green region is excluded by catalysis of ${ }^{6} \mathrm{Li}$ production [41. We also show the region which would be excluded solely by free streaming arguments. The dotted lines show the slepton lifetime. In the presence of a gravitino with $M_{\tilde{G}}=100 \mathrm{GeV}$ the slepton would dominantly decay into the hidden $U(1)$ gaugino, except in the red lower region. The dashed lines show the region that is potentially excluded by bounds on $\Lambda C W D M$ models in scenarios where the thermally produced hidden $U(1)$ gaugino decays into a gravitino LSP with large free streaming length (see Fig. (4). The lines correspond to a dark matter fraction $f=0.02$ and $f=0.2$ which is allowed to be warm. 


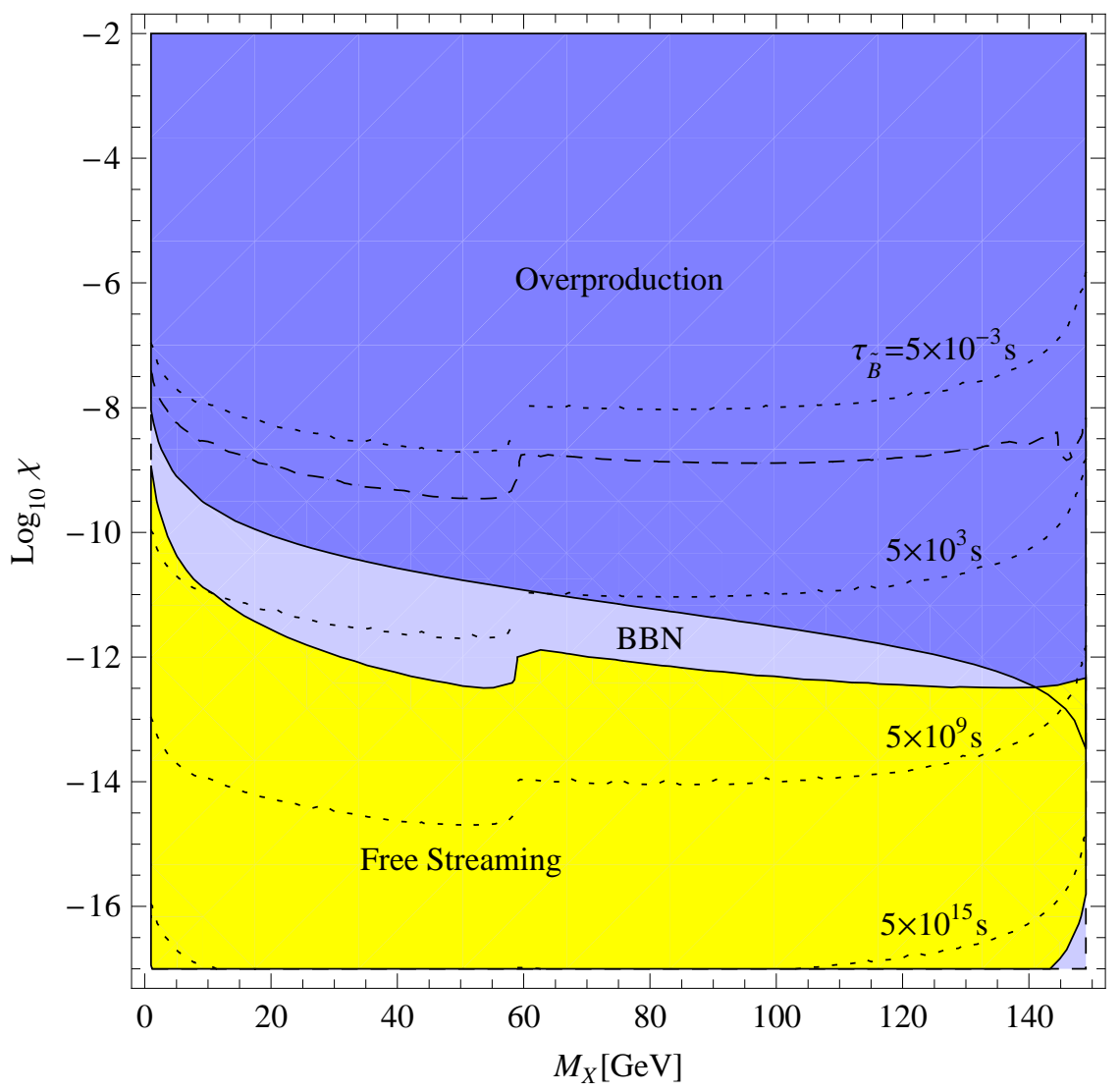

Figure 2: Summary of bounds on the hidden $U(1)$ gaugino parameter space for the case of a bino-like neutralino NLSP. We take $M_{B}=150 \mathrm{GeV}$. The dark blue region is excluded by thermal overproduction. Below this region, the hidden $U(1)$ gaugino would be dominantly produced via late decaying neutralinos. This scenario is totally excluded by BBN [40] (light blue region). The bound actually strongly overlaps with the overproduction region (dashed line). We also show the region (in yellow) that would be solely excluded by free streaming arguments. The dotted lines show the lifetime of the neutralino. (We used $M_{\mathrm{sf}}=400 \mathrm{GeV}$ and $\mu=300 \mathrm{GeV}$ for the branching ratios). 


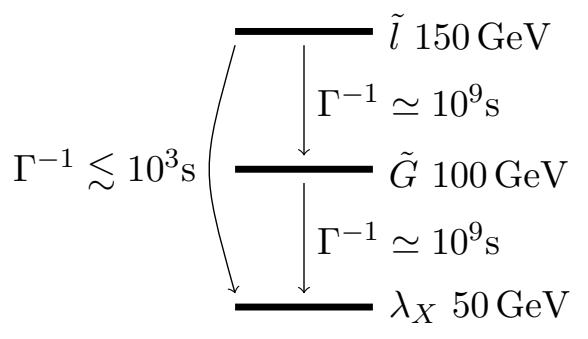

a

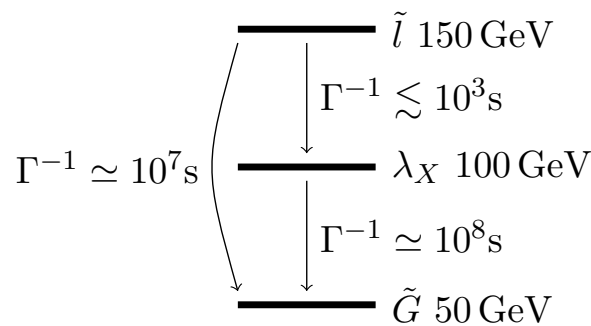

b

Figure 3: Spectra for (a) gravitino NLSP and (b) gravitino LSP (cf. last two cases in Tab. 2). We show the widths for the different decay processes for typical particle masses. The mixing parameter is assumed to lie in the allowed region of Fig. 1]

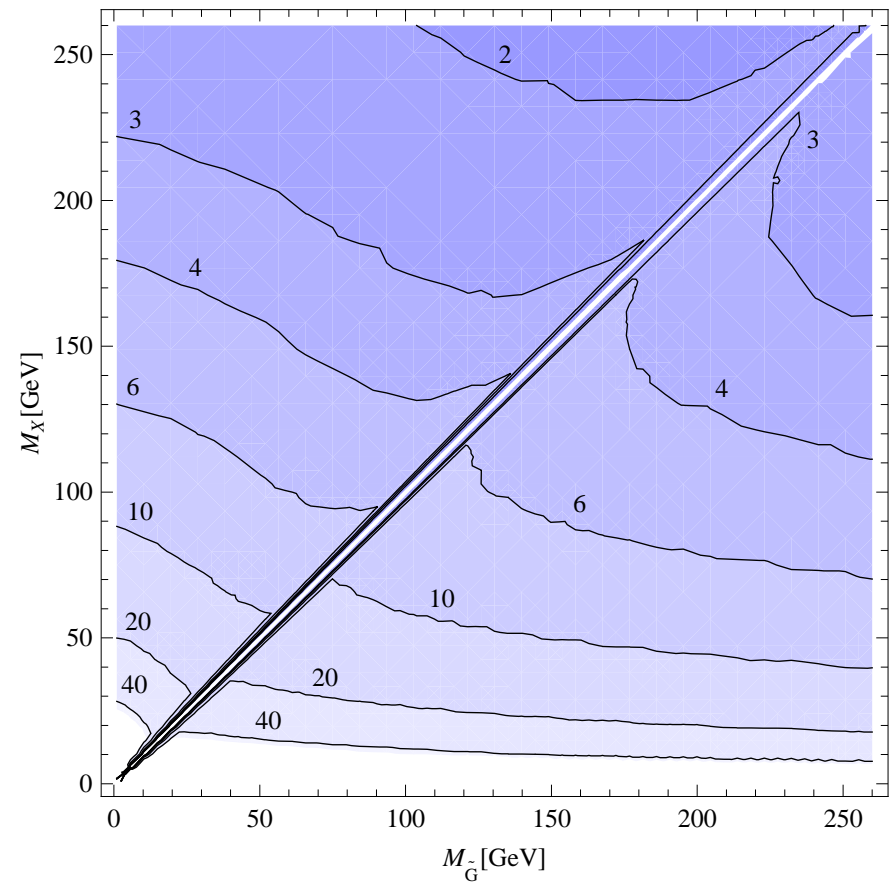

Figure 4: Contour plot of free streaming lengths in units of Mpc. The upper left (lower right) corner shows the free streaming length of gravitinos (hidden $U(1)$ gauginos) that stem from the late decay of hidden $U(1)$ gauginos (gravitinos). 


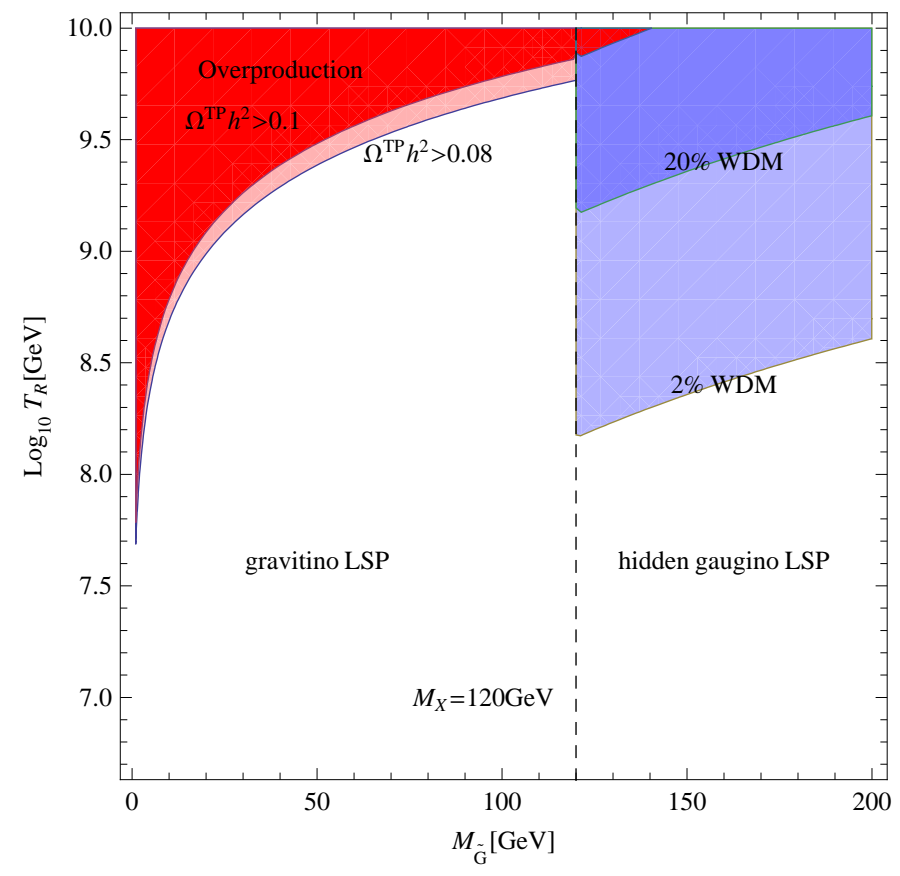

Figure 5: Bounds on the reheating temperature as function of the gravitino mass, using Eq. (18) with $m_{\tilde{g}}=800 \mathrm{GeV}$. The mass of the hidden $U(1)$ gaugino is fixed to $M_{X}=120 \mathrm{GeV}$. If the gravitino is the LSP (left part), the reheating temperature is only bounded by overproduction arguments (dark red region), which are only slightly strengthened when, say, $20 \%$ of the gravitino abundance is due to non-thermal production (light red region). A gravitino NLSP (right part) would late decay into the hidden $U(1)$ gaugino, yielding a warm dark matter component. If only a fraction of $20 \%$ or $2 \%$ of dark matter is allowed to be warm (with free streaming lengths as shown in Fig. 4), the corresponding blue regions are excluded. 


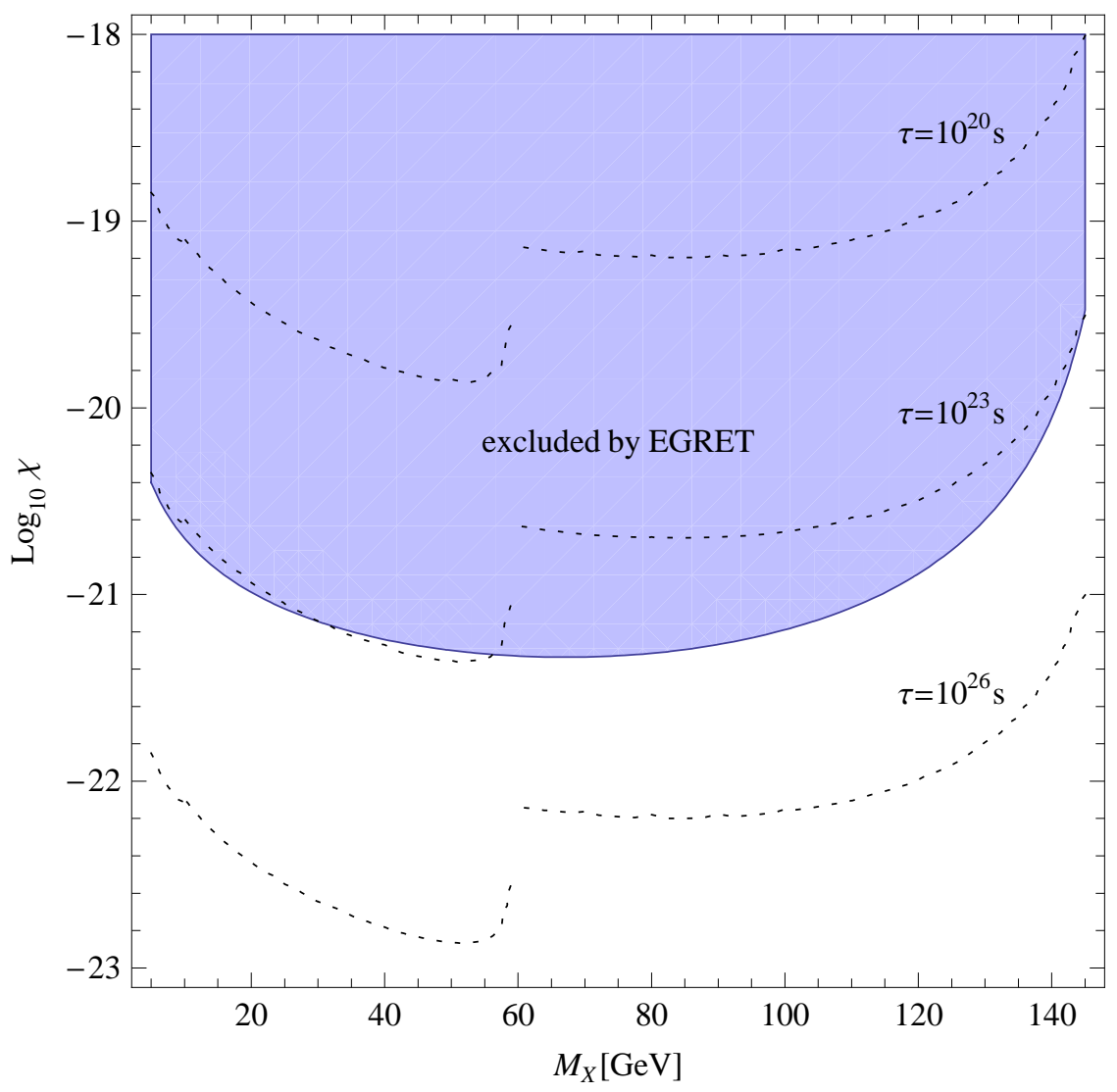

Figure 6: Region of the hidden $U(1)$ gaugino parameter space which is excluded by confronting the EGRET measurements [73] with the extragalactic gamma ray flux produced in the decay $\lambda_{B} \rightarrow \gamma \lambda_{X}$ (blue region). The bino mass is fixed to $M_{B}=150 \mathrm{GeV}$. We also show for reference the isocurves for the neutralino life-time. 